\title{
ARTIGOS
}

Recebido em 23.12.2011. Aprovado em 05.11.2012

Avaliado pelo sistema double blind review. Editor Científico: Dario de Oliveira Lima Filho

\section{COMO ENTENDER A VAIDADE FEMININA UTILIZANDO A AUTOESTIMA E A PERSONALIDADE}

\author{
How to understand women's vanity using self-steem and personality \\ Cómo entender la vanidad femenina utilizando la autoestima y la personalidad
}

\section{RESUMO}

O artigo busca compreender a relação da autoestima e variáveis de personalidade na vaidade feminina e como essas associações podem influenciar a propensão de buscar cirurgias plásticas estéticas (CPEs). Usando o modelo 3M (MOWEN, 2000), realizou-se um levantamento com 697 alunas de uma universidade federal brasileira, com idade entre 18 e 50 anos. Vaidade foi operacionalizada por dois traços: preocupação com aparência, que consiste em uma observação exagerada da aparência física, e visão vaidosa, referente à avaliação excessivamente positiva da própria aparência. Os principais achados indicam que quanto maior a criatividade, extroversão, amabilidade e necessidade de manter e de realçar o corpo, maior será a autoestima, e quanto maior a autoestima, menor a preocupação exagerada com a aparência física e maior a avaliação positiva que uma mulher faz de sua aparência. Mulheres materialistas e com necessidade maior de recursos corporais são mais excessivamente preocupadas com a aparência. A influência da vaidade na propensão à CPE deu-se por meio do traço preocupação com aparência.

PALAVRAS-CHAVE | Personalidade, autoestima, vaidade, cirurgia plástica, modelo 3M.

\begin{abstract}
The article seeks to understand the relationship between self-esteem and personality traits in feminine vanity and how these associations may influence propensity to undergo into cosmetic surgery. The subject has been studied using the 3M model (Mowen, 2000). The research is based on a survey of 697 students in a Brazilian Federal university, aged between 18 and 50 years old. Vanity was operationalized by two traits: vanity concern (an exaggerated concern about physical appearance), and vanity view (an excessively positive evaluation of one's own appearance). The main finding indicate that a greater creativity, extroversion, kindness and need of body resources, is related to greater self-esteem, and also that the greater their self-esteem, the less vanity concern and the greater the vanity view. Women who are materialistic and have a greater need to body resources are more excessively concerned with their appearance. The need of body resources is also positively related to vanity view. The influence of self-esteem in the propensity to plastic surgery was noticed by means of vanity concern.
\end{abstract}

KEYWORDS / Personality, self-esteem, vanity, cosmetic surgery, 3M model.

\section{RESUMEN}

CÁTIA FABÍOLA PARREIRA DE AVELAR catiaavelar@yahoo.com.br Professora do Departamento de Ciências Administrativas, Universidade Federal de Minas

Gerais - Belo Horizonte - MG, Brasil

\section{RICARDO TEIXEIRA VEIGA}

ricardo.necc@gmail.com

Professor do Departamento

de Ciências Administrativas,

Universidade Federal de Minas

Gerais - Belo Horizonte - MG, Brasil
El artículo trata de comprender la relación de la autoestima y variables de personalidad en la vanidad femenina y como esas asociaciones pueden influir en la propensión de buscar cirugías plásticas estéticas (CPES). Usando el modelo 3M (MOWEN, 2000), se realizó un estudio con 697 alumnas de una universidad federal brasileña, con edad entre 18 y 50 años. Vanidad fue determinada por dos rasgos: preocupación con la apariencia, que consiste en una observación exagerada de la apariencia física y la visión vanidosa, referente a la evaluación excesivamente positiva de la propia apariencia. Los principales resultados indican que cuanto mayor es la creatividad, extroversión, amabilidad y necesidad de mantener y de destacar el cuerpo, mayor será la autoestima y cuanto mayor es la autoestima, menor es la preocupación exagerada con la apariencia física y mayor es la evaluación positiva realizada por una mujer respecto a su apariencia. Mujeres materialistas y con necesidad mayor de recursos corporales son más excesivamente preocupadas con la apariencia. La influencia de la vanidad en la propensión a CPE ocurrió por medio del rasgo preocupación con la apariencia.

PALABRAS CLAVE I Personalidad, autoestima, vanidad, cirugia plastica, modelo 3M. 


\section{INTRODUÇÃO}

Mudanças estruturais, como o aumento do número de pessoas do sexo feminino trabalhando, competição e discriminação no local de trabalho, estimulam as mulheres a ficarem mais vaidosas e com maior medo de envelhecer, levando-as ao consumo de produtos e serviços para aumentar sua competitividade no mercado de trabalho e nas relações sociais (EDMONDS, 2002). De fato, indivíduos avaliam sua aparência, baseando-se no que a sociedade considera atraente, significativo e valioso. Logo, as sensações de um indivíduo na avaliação de si mesmo (e, consequentemente, sua autoestima) podem ser afetadas pelo modo como uma pessoa acredita que a sociedade valoriza seu corpo (RICCIARDELLI e CLOW, 2009). Segundo Bauman (2008), apesar da sensação de poder "escolher" oferecida pela sociedade de consumo, todas as opções são pré-selecionadas, pré-certificadas e pré-escritas pela mídia, ou seja, um corpo que se assemeIha àqueles expostos e avaliados como "desejáveis" leva sua possuidora a ter maior satisfação e autoestima.

Abdala (2004) explica que os inúmeros produtos e serviços que trazem a promessa de tornar as pessoas mais belas são utilizados para gerenciar a impressão que a aparência causa nos outros, uma vez que se buscam, conscientemente ou não, os benefícios associados com a beleza (por exemplo, elogios e conquistas amorosas) e a redução da insatisfação com a própria imagem ou autoconceito. As recompensas sociais obtidas com base na beleza servem como motivadores para a exacerbação da vaidade física no mundo contemporâneo. A consequência do consumismo de produtos estéticos, associado à vaidade física, pode ser extremada ou equilibrada, dependendo do nível de envolvimento das pessoas com os produtos, demandas e recompensas sociais relativas a esse processo.

Para evitar sentimentos negativos, como a insatisfação e a baixa autoestima, as opções de embelezamento disponíveis no mercado compreendem cosméticos, exercícios, tratamentos estéticos, dietas e CPEs. Strehlau, Claro e Neto (2010), em um estudo realizado numa capital brasileira, mostram que quanto maior o grau de vaidade da mulher, maior a utilização de cosméticos, tratamentos domésticos de beleza e propensão para a realização de procedimentos cirúrgicos. Segundo a Associação Brasileira da Indústria de Higiene Pessoal, Perfumaria e Cosméticos (ASSOCIAÇÃO..., 2010), o Brasil é o terceiro maior mercado do setor (atrás dos Estados Unidos e do Japão), mantendo $8 \%$ de participação no mercado mundial e $53 \%$ de participação do mercado da América Latina, no ano de 2009. Já um relatório da Sociedade Brasileira de Cirurgia Plástica (SBCP) (SOCIEDADE..., 2009) indica que, de setembro de 2007 a dezembro de 2008, foram realizadas, no País, por profissionais especializa- dos e membros da SBCP, aproximadamente 459 mil CPEs, e, em $88 \%$ dos casos, os pacientes eram mulheres. A legislação brasileira não exige o título de especialista em cirurgia plástica para os médicos que realizam CPEs, o que sugere que esse número, na realidade, pode ser bem maior, já que muitos médicos sem especialização em CPE também realizam esse tipo de cirurgia.

A personalidade tem sido um campo árido e pouco explorado pelo marketing (BAUMGARTNER, 2002), porém Mowen (2000) propôs o modelo metateórico de motivação e personalidade (3M), visando resgatar o debate sobre a relação personalidade-consumo, de modo mais abrangente e com maior potencial de aplicação prática que estudos anteriores. Por se tratar de um modelo bem-sucedido, optou-se por ele nesta pesquisa, para investigar os antecedentes de personalidade da busca por CPEs.

0 conhecimento dos fatores de personalidade que influenciam a busca por CPEs pode ter várias aplicações gerenciais, tais como: (1) segmentação - um número suficiente de pessoas que possuam traços de personalidade e motivações semelhantes pode representar um mercado-alvo atraente para empresas de produtos e serviços de embelezamento; (2) desenvolvimento de mensagens mais eficazes - a descoberta dos antecedentes de personalidade correlacionados à propensão de realizar CPEs pode apoiar a elaboração de campanhas tanto para estimular o consumo quanto para reduzi-lo; (3) posicionamento - características e atributos consistentes com os traços de personalidade relevantes para explicar a disposição em fazer CPEs podem ser destacados no posicionamento de ofertas e marcas.

\section{BELEZA, AUTOESTIMA E VAIDADE}

Para aumentar a compreensão da centralidade da beleza no cotidiano, Sarwer, Magee e Clark (2004) fizeram revisão bibliográfica de estudos fisiológicos e socioculturais sobre o papel da aparência física no cotidiano e sua influência potencial na decisão de procurar tratamentos médicos cosméticos, mostrando que: (1) as características físicas da beleza desempenham um papel significante na seleção de parceiros românticos e sexuais entre humanos; (2) preferências de características físicas parecem ser condicionadas pela exposição a padrões sociais de beleza; (3) são atribuídas características de personalidade mais positivas a indivíduos atraentes; (4) indivíduos atraentes frequentemente recebem tratamento favorável, em várias interações sociais.

Muitas vezes, a beleza é reduzida a traços físicos específicos - pele clara, olhos vivos, cabelo brilhante etc. Essas ca- 
racterísticas, frequentemente, servem de marcadores de outras mais sutis, tais como juventude, resistência a doenças, simetria, proporções de corpo e medidas, que comunicam sinais de saúde e potencial reprodutivo.

Para citar exemplos de como a atratividade interfere nas relações humanas, Sarwer, Magee e Clark (2004) identificaram estudos que mostraram que: (1) a percepção dos adultos sobre crianças e a autopercepção delas é influenciada por sua aparência física; (2) julgamentos da atratividade física influem na percepção de estudantes a respeito uns dos outros e na relação entre estudantes e professores, poisprofessores tendem a perceber belos estudantes como tendo mais habilidades, confiança, inteligência e potencial acadêmico do que estudantes pouco atraentes; (3) belas crianças são mais populares do que os seus pares pouco atraentes; (4) candidatos mais atraentes têm maior probabilidade de receber ofertas de emprego; (5) indivíduos bonitos parecem ter maior probabilidade de receber ajuda e menor probabilidade de receber pedidos de ajuda; e (6) quase todos os homens e mulheres testados apresentaram preferências pelos parceiros fisicamente mais atraentes entre os disponíveis, levando à conclusão de que a atração física provavelmente, inicie a maior parte de relações românticas, embora, em situações reais, características de personalidade que atraem e geram confiança em outras pessoas, tais como inteligência, senso de humor e lealdade, desempenhem papel mais central nessas relações.

Esses dados indicam que beleza física traz benefícios, pois os indivíduos fisicamente atraentes são julgados mais positivamente por outros, além de receberem tratamento preferencial em numerosos encontros interpessoais durante a vida. Mesmo em situações em que seria desejável que a aparência não importasse, pesquisas sugerem que ela realmente conta.

0 padrão de beleza pode variar de acordo com o contexto histórico e cultural. Os membros de uma sociedade buscam emular a beleza ideal por meio da "imitação prestigiosa: os indivíduos imitam atos, comportamentos e corpos que obtiveram êxito e que viram ser bem-sucedidos" (MAUSS, 1974 apud GOLDENBERG, 2005, p. 68).

Autoestima pode se concebida como uma avaliação que a pessoa faz de si mesma, a qual implica um sentimento de valor, que engloba um componente predominantemente afetivo, expresso numa atitude de aprovação/desaprovação em relação a si própria (ROSENBERG, 1965 apud ROMANO, NEGREIROS, MARTINS, 2007). Strehlau, Claro e Neto (2010) descobriram que quanto maior o grau de vaidade da mulher, maior a relação entre sua autoestima e o corpo.

Etcoff e outros (2004) exploraram empiricamente 0 que "beleza" significa para as mulheres. O estudo foi reali- zado em 10 países (Estados Unidos, Canadá, Inglaterra, Itália, França, Portugal, Escócia, Brasil, Argentina e Japão), com 3.300 mulheres, com entre 18 e 64 anos, entre fevereiro e março de 2004. Os resultados mostram que: (1) as mulheres estão menos satisfeitas com sua beleza do que com todas as dimensões de sua vida, exceto sucesso financeiro; (2) aparência física influencia a forma como se sentem a respeito de si mesmas, a avaliação da beleza própria e dos outros; e (3) a maioria das participantes gostaria de se sentir e ser percebida como bela. Quase metade (48\%) das mulheres que participaram do estudo concordou plenamente com a frase: "quando me sinto menos bonita, me sinto pior em relação a mim mesma”, o que ilustra o impacto da avaliação da beleza na autoestima. Mais da metade (68\%) das mulheres concordou plenamente que a mídia e os anúncios mostram padrões de beleza irreais e que a maioria das mulheres nunca os alcançará, e dados da pesquisa mostraram que os ideais de beleza, em termos de aparência física, levam muitas mulheres a se esforçarem para alcançá-los. Dado que, muitas vezes, esses ideais são inatingíveis - seja a magreza das modelos ou as formas voluptuosas alcançadas por musculação pesada -, as mulheres apresentam dificuldades em se considerarem belas (apenas $2 \%$ da amostra global se descreveram como tendo aparência física “bela”).

Edmonds (2002), Ricciardelli e Clow (2009), Abdala (2004) e Strehlau, Claro e Neto (2010) indicam que, possivelmente, grande parte da receita da indústria da beleza provém de mulheres vaidosas. Netemeyer, Burton e Lichtenstein (1995) definiram vaidade como composta por quatro traços: preocupação excessiva com a aparência, visão positiva (e exagerada) da aparência física, preocupação excessiva com a realização e alcance de metas e a visão positiva (e às vezes exagerada) da realização dessas metas. Mowen, Longoria e Sallee (2009) utilizaram duas das escalas desenvolvidas por Netemeyer, Burton e Lichtenstein (1995) para avaliar a vaidade: preocupação com a aparência (vanity concern), que se refere à observação exagerada da aparência física, e visão vaidosa (vanity view), que se refere à visão exageradamente positiva da aparência física de uma pessoa. Esses dois traços foram tratados separadamente como dois construtos independentes, baseando-se em resultados do estudo de Netemeyer, Burton e Lichtenstein (1995). A vaidade, então, é tratada neste artigo apenas com base em seus aspectos relacionados à aparência física, pois se acredita que essas dimensões da vaidade motivam o consumo de produtos voltados para melhorar a aparência e autoestima. Por isso, vaidade é definida como "preocupação excessiva com a aparência física e avaliação excessivamente positiva da própria aparência”. 


\section{PERSONALIDADE E COMPORTAMENTO DO CONSUMIDOR}

Seguindo-se Mowen (2000), interpretou-se personalidade como os padrões distintivos de comportamento que caracterizam a herança genética singular e adaptação de cada indivíduo às situações de sua vida. 0 objetivo das pesquisas com o consumidor que utilizam a personalidade é identificar as variáveis que distinguem grupos de consumidores (MOWEN, 2000; MOWEN e MINOR, 2003). Segundo Mowen e Minor (2003), Engel, Blackwell e Miniard (2000) e Sheth, Mittal e Newman (2001), as abordagens teóricas dominantes no estudo da personalidade, no campo do comportamento do consumidor, são a psicanalítica e a de traço (ou características), em que as personalidades são tratadas como combinações singulares de traços básicos.

Nos primeiros anos, dois problemas com a pesquisa de traços de personalidade no campo do comportamento do consumidor eram: (1) nenhuma escala de personalidade havia sido desenvolvida especialmente para mensurar comportamento de consumo e (2) os pesquisadores não consideravam quais traços de personalidade seriam importantes para as decisões de consumo (SHETH, MITTAL, NEWMAN, 2001). Devido a esses problemas, estudos anteriormente feitos por pesquisadores do consumo, empregando a abordagem dos traços, foram criticados por serem fracos e inconclusivos (MOWEN e MINOR, 2003; ENGEL, BLACKWELL, MINIARD, 2000). Além disso, uma excessiva quantidade de traços foi identificada, tanto por pesquisadores do consumidor como por psicólogos, sem um princípio organizador subjacente.

Desafiando esse quadro, Mowen (2000) desenvolveu uma nova abordagem para se compreender o impacto dos traços de personalidade no comportamento do consumidor, denominada modelo metateórico de motivação e personalidade (3M). Vários estudos têm sido feitos, nos últimos 10 anos, utilizando o $3 \mathrm{M}$, para explicar uma grande diversidade de comportamentos de consumo, por exemplo: Mowen (2004), que estudou a competitividade e sua consequência no comportamento de consumo; Mowen e Sujan (2005), que estudaram o comportamento de voluntariado; Basso e outros (2009), que usaram o modelo 3M para compreensão da lealdade; Monteiro, Veiga e Gonçalves (2009), que investigaram a participação em esportes e hábitos de moda; Ribeiro e Veiga (2010), que elaboraram escala para medir o consumo sustentável. Tais estudos mostram que uma boa parte da variação comportamental pode ser atribuída à estrutura hierárquica de traços de motivação e personalidade proposta pelo modelo.

No modelo 3M, traço de personalidade é definido como

“qualquer construto intrapsíquico que pode ser mensurado de forma válida e confiável e que prevê diferenças individuais em sentimentos, pensamentos e comportamento" (MOWEN, 2000, p. 2). Personalidade é definida como um "conjunto hierarquicamente relacionado de construtos intrapsíquicos (traços) que revelam consistência no transcorrer do tempo e que se combinam com o contexto para influenciar sentimentos, pensamentos e comportamentos dos indivíduos" (MOWEN, 2000, p. 2). 0 modelo fundamenta-se nos princípios da teoria do controle, psicologia evolucionária, personalidade hierárquica e no modelo de cinco fatores da personalidade. Segundo o autor, a estrutura de traços de personalidade pode ser organizada segundo níveis crescentes de abstração, partindo de comportamentos que podem ser facilmente observados até os traços elementares, que são abstratos e abrangentes. Mowen (2000) utiliza quatro níveis hierárquicos de traços de personalidade em seu modelo, sendo eles: traços elementares, traços compostos, traços situacionais e traços superficiais.

O nível mais básico é o dos traços elementares que resultam da genética e do aprendizado anterior. No modelo, são propostos oito traços elementares, sendo esses: amabilidade, extroversão, abertura a experiências, neuroticismo (ou instabilidade emocional), organização, necessidade de recursos materiais, necessidade de recursos corporais e necessidade de excitação. O Quadro 1 mostra a definição de cada um desses traços. Os traços elementares combinam-se com processos culturais e com o aprendizado anteriormente vivido para compor os traços compostos como autoestima. Traços compostos combinam com o contexto do comportamento para produzir os traços situacionais, que são as diferenças individuais expressadas dentro de um contexto geral, como interesse por esportes, motivação para saúde, vaidade. Os traços situacionais combinam com atitudes permanentes e envolvimento quanto a classes de comportamentos/produtos/serviços para criar traços superficiais, que representam tendências duráveis de atuar a respeito de categorias de produtos/serviços ou um domínio específico do comportamento. Traços superficiais podem ser definidos como disposição duradoura para agir em contextos específicos, como propensão a fazer cirurgia plástica, adotar dietas saudáveis ou consumir responsavelmente.

No modelo $3 \mathrm{M}$, a personalidade age como um moderador do modo de agir e consumir dos indivíduos, pois estímulos que incentivam indivíduos caracterizados por um traço de personalidade podem desestimular indivíduos não caracterizados por esses traços. Mowen (2000) sugere que uma comunicação pode alterar a percepção que indivíduos têm do seu estado atual de satisfação. Se, por exemplo, o consumo de cosméticos, tratamentos domésticos de beleza e propensão para a realização de procedimentos cirúrgicos é influenciado pela vaida- 
de (preocupação exagerada e visão excessivamente positiva da aparência física) e vaidade associa-se à autoestima e necessidade de recursos corporais, então uma comunicação que aborde pessoas felizes, com elevada autoestima e corpos bem delineados, poderia motivar pessoas mais vaidosas a consumirem produtos e serviços de embelezamento, ao passo que poderia desestimular pessoas menos vaidosas a terem o mesmo comportamento.

\section{Quadro 1. Definições dos oito traços elementares do modelo 3M}

\begin{tabular}{l|l}
\hline Traço elementar & Definição \\
\hline Abertura a experiências & Necessidade de encontrar novas soluções, expressar ideias originais e usar a imaginação na execução de tarefas. \\
\hline Organização & Necessidade de ser organizado, ordenado e eficiente na realização de tarefas. \\
\hline Extroversão & Operacionalizado como oposto de introversão (tendência de vivenciar sentimentos de timidez). \\
\hline Amabilidade & Necessidade de expressar bondade e simpatia pelas pessoas. \\
\hline Neuroticismo & Tendência à emotividade expressa por mau humor e atitude temperamental. \\
\hline Necessidade de recursos materiais & Necessidade de reunir e possuir bens materiais. \\
\hline Necessidade de excitação & Desejo por estimulação e excitação. \\
\hline Necessidade de recursos corporais & Necessidade de manter e realçar o corpo. \\
\hline
\end{tabular}

Fonte: Adaptado de Mowen, 2000.

\section{HIPÓTESES DE PESQUISA}

Nas aplicações do modelo 3M, as hipóteses de relacionamento entre os traços de personalidade são elaboradas com base em estudos prévios e análise lógica, considerando-se as definições dos construtos e a provável correlação dos traços mais abstratos com traços mais concretos (MOWEN, 2000).

Em seus estudos sobre a autoestima, Mowen (2000) descobriu que o construto autoestima pode ser considerado traço composto no modelo 3M. Swami e coautores (2009) descobriram que a autoestima está intimamente ligada com a avaliação da atratividade, que pode ser considerada como vaidade. Markey e Markey (2009) mostraram que as mulheres insatisfeitas com os seus corpos estiveram mais interessadas em CPE do que mulheres que estavam relativamente satisfeitas com os seus corpos. Como autoestima relaciona-se com a avaliação que uma pessoa faz de si, visão vaidosa engloba avaliação da própria aparência física e preocupação com a aparência relaciona-se com o quanto alguém se preocupa exageradamente com sua própria aparência, pode-se deduzir, com base nos dados apresentados, que quanto pior a autoestima de uma pessoa, maior a probabilidade de essa pessoa avaliar como ruim sua aparência, preocupar-se mais em melhorá-la e buscar produtos ou serviços de embelezamento para aumentar sua autoestima.

Assim sendo, antecipa-se que a autoestima se associa negativamente com preocupação com a aparência, uma vez que pessoas com baixa autoestima parecem preocupar-se mais com a aparência, e associa-se positivamente com visão vaidosa, já que pessoas que apresentam uma atitude positiva em relação a si acabam por ter a mesma atitude em relação a sua aparência física. Vários estudos mostraram, também, que a autoestima está associada à propensão à CPE (EDMONDS, 2002, 2007; ASKEEGARD, GERSTEN, LANGER, 2002; GIMLIN, 2000), sendo a CPE buscada por pessoas que queriam melhorar sua autoestima. Esses resultados levam à primeira hipótese de pesquisa:

H1: Autoestima está negativamente relacionada com preocupações com a aparência (a) e propensão à CPE (b), e positivamente relacionada com visão vaidosa (c).

Mowen (2000) descobriu relações entre autoestima e os seguintes traços elementares: neuroticismo, extroversão, necessidade de recursos corporais, organização, necessidade de excitação, amabilidade e abertura a experiências (somente a relação com o traço elementar necessidade de recursos materiais não foi significante em seu estudo). Com suporte desses dados, foi formulada a seguinte hipótese.

$\mathrm{H} 2$ : Autoestima relaciona-se com neuroticismo (a), extroversão (b), necessidade de recursos corporais (c), organização (d), necessidade de excitação (e), amabilidade (f) e abertura a experiências (g).

Sobre os traços elementares, Mowen, Longoria e Sallee (2009) mostraram que o traço preocupação com a aparência associou-se positivamente com os traços necessidade de recur- 
sos materiais e necessidade de recursos corporais e que o traço visão vaidosa associou-se positivamente com necessidade de recursos corporais. Foram descobertas, ainda, as seguintes relações no estudo: neuroticismo (instabilidade emocional) e necessidade de recursos materiais associaram-se positivamente com a propensão de se submeter à CPE, enquanto o traço organização associou-se negativamente com propensão à CPE. Esses dados dão origem a novas hipóteses:

$\mathrm{H}_{3}$ : Necessidade de recursos corporais está positivamente relacionada com preocupações com a aparência (a), visão vaidosa (b) e propensão à CPE (c).

H4: Necessidade de recursos materiais está positivamente associada com preocupações com a aparência (a) e propensão à CPE (b).

$\mathrm{H}_{5}$ : Propensão à CPE relaciona-se positivamente com neuroticismo (a) e negativamente com organização (b).

Mowen, Longoria e Sallee (2009) descobriram que a vaidade - operacionalizada pelos traços preocupação com a aparência e visão vaidosa - relacionou-se com a propensão à CPE. No Brasil, Strehlau, Claro e Neto (2010) também descobriram uma relação entre vaidade e busca por CPE. Davis (2002) notou que para mulheres, a falta da autoconfiança, a sensação de ser diferente e preocupações sobre a aparência foram identificadas como as razões principais para escolher uma CPE. Os estudos fornecem evidências para a formulação de mais duas hipóteses:

H6: Preocupação com a aparência física está positivamente relacionada com propensão à CPE.

H7: Visão vaidosa está negativamente relacionada com propensão à CPE.
A Figura 1 mostra as relações hipotéticas entre traços elementares, compostos, situacionais e superficiais. Evidências de estudos mostram que nem todos os traços possuem uma relação estaticamente significante e que traços do mesmo nível hierárquico podem se correlacionar.

\section{PROCEDIMENTOS METODOLÓGICOS}

Para testar as hipóteses e o modelo proposto no presente estudo, foi aplicado um questionário impresso e estruturado com perguntas fechadas a 697 alunas dos cursos de graduação e pósgraduação de uma universidade federal em uma capital brasileira, no mês de novembro de 2010. Assim, trata-se de um estudo transversal feito com uma amostra não probabilística de conveniência. Na análise dos dados, realizou-se procedimento de validação cruzada (cross-validation), subdvidindo-se a amostra em duas subamostras, de modo a realizar a análise fatorial exploratória numa delas e o exame do modelo teórico e hipóteses na outra subamostra, utilizando-se modelagem de equações estruturais, conforme recomendação de Hair e outros (2010).

Monteiro (2008) utilizou-se de itens semelhantes aos citados por Mowen, Longoria e Sallee (2009) nas escalas para mensurar traços elementares, em seu estudo sobre inovação em moda com estudantes universitários no contexto brasileiro. Sua versão dos itens em português da escala de Mowen (2000) apresentou boa confiabilidade e validade. Por esse motivo, foi utilizada a adaptação de Monteiro (2008) para mensurar os traços citados. Para responder a cada item das escalas, as respondentes deveriam marcar, em uma escala de 7 pontos, sendo $1=$ "nunca" e 7 = "sempre", a melhor opção para a pergunta: "Com que frequência você se sente ou age dessa forma?".

Figura 1. Estrutura hierárquica parcial e hipotética de traços do modelo 3M relacionados com a propensão à CPE

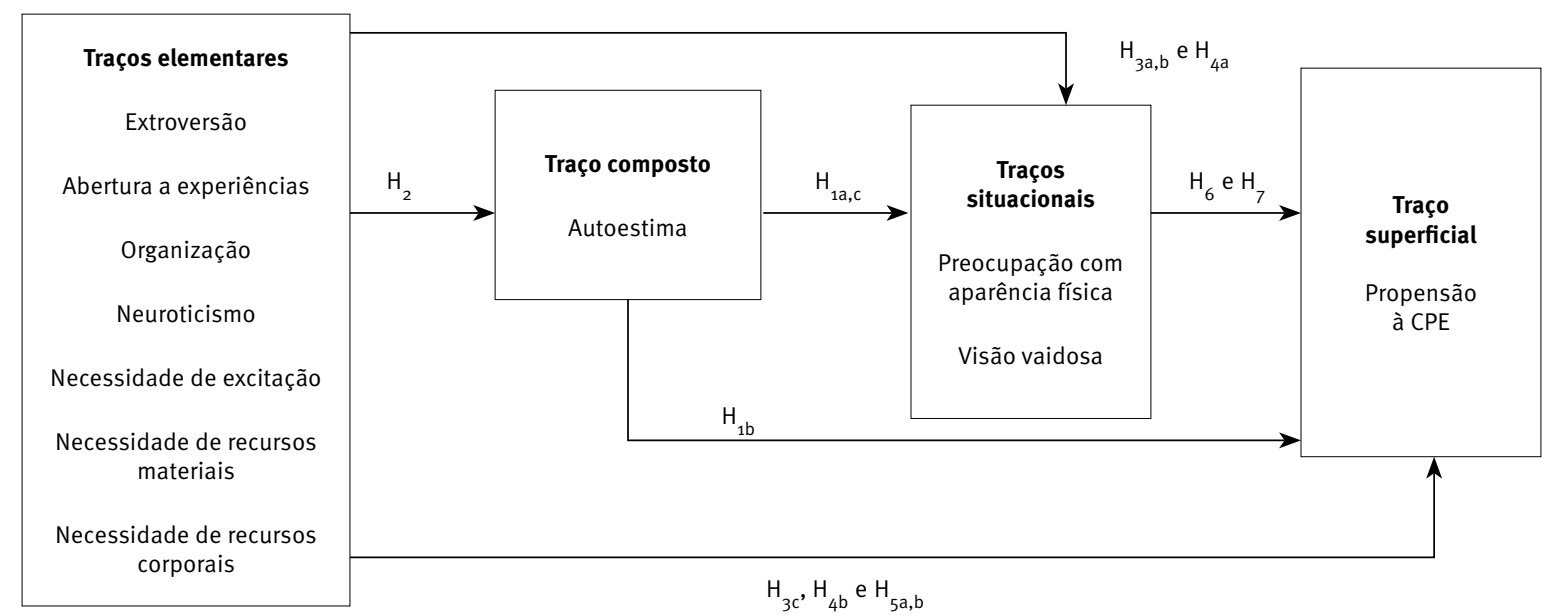


Os itens para mensuração do traço composto autoestima foram retirados dos estudos de Mowen (2000), os quais apresentaram evidência de validade e confiabilidade. A mensuração dos traços situacionais (preocupação com aparência e visão vaidosa) foi feita no estudo de Mowen, Longoria e Sallee (2009) com base em itens retirados do trabalho de Netemeyer, Burton e Lichtenstein (1995). Os mesmos itens foram utilizados nesta pesquisa, por apresentarem boa confiabilidade e validade.

0 traço superficial propensão à CPE foi medido no estudo de Mowen, Longoria e Sallee (2009) por meio de quatro perguntas. Etcoff e outros (2004) descobriram em seu estudo que, quando perguntadas sobre se queriam fazer algum tipo de CPE, 23\% das mulheres pesquisadas disseram que sim; porém, quando questionadas sobre se fariam o procedimento, se tivessem certeza de que era seguro e de graça, o número de mulheres que responderam positivamente à pergunta subiu para $40 \%$, principalmente no Brasil, Argentina e Esta- dos Unidos. Esse resultado levou à inclusão de mais duas perguntas na escala sobre propensão à CPE: "Eu me submeteria à cirurgia plástica estética, se fosse de graça” e "Eu me submeteria à cirurgia plástica estética, se tivesse a certeza de que o procedimento é seguro". Não foi encontrada adaptação para os itens das escalas dos traços situacionais, superficial e do traço composto para populações no contexto brasileiro. Os itens originais em inglês foram traduzidos para o português. Procedimentos de tradução reversa retornaram a escala traduzida à versão original, e não foram encontradas diferenças significativas. Traduções alternativas e adaptações foram produzidas para preservar o sentido original dos indicadores. Cada item foi avaliado por escalas de sete pontos do tipo Likert, sendo 1 = "discordo totalmente" e 7 = "concordo totalmente". Um resumo das fontes que foram utilizadas para compor as escalas do questionário é apresentado no Quadro 2.

\section{Quadro 2. Fonte de itens das escalas}

\begin{tabular}{l|l|l}
\hline Escala & Traço & Fonte \\
\hline Extroversão & Elementar & Mowen (2000); Monteiro (2008) \\
\hline Amabilidade & Elementar & Mowen (2000); Monteiro (2008) \\
\hline Neuroticismo & Elementar & Mowen (2000); Monteiro (2008) \\
\hline Organização & Elementar & Mowen (2000); Monteiro (2008) \\
\hline Necessidade de recursos corporais & Elementar & Mowen (2000); Monteiro (2008) \\
\hline Necessidade de recursos materiais & Elementar & Mowen (2000); Monteiro (2008) \\
\hline Necessidade de excitação & Elementar & Mowen (2000); Monteiro (2008) \\
\hline Autoestima & Superficial & Mowen (2000) \\
\hline Preocupação com aparência & Situacional & Netemeyer, Burton, Lichtenstein (1995) \\
\hline Visão vaidosa & Situacional & Mowen, Longoria, Sallee (2009) \\
\hline Propensão à cirurgia plástica estética & Superficial & Etcoff e outros (2004); Mowen, Longoria, Sallee (2009) \\
\hline
\end{tabular}

Os itens foram pré-testados com 57 indivíduos, levando a pequenos ajustes de fraseados. Cabe destacar que o instrumento de pesquisa fazia parte de um estudo maior. Após refinamento, um questionário com 69 questões foi aplicado a uma amostra de conveniência $(n=697)$. A amostra foi composta por $94,4 \%$ de alunas de cursos de graduação e o restante, por alunas de pós-graduação. Mais da metade $(58,1 \%)$ das respondentes tinha de 21 a 30 anos. 0 segundo grupo etário com mais alunas foi o de 18 a 20 anos, que correspondeu a 31,6\% da amostra; o restante das respondentes tinha mais de 31 anos. Quanto à renda familiar, a maior parte das famílias das alunas da amostra $(56,6 \%)$ ganhava até $R \$ 3.100,00$ por mês ou, aproximada- mente, seis salários mínimos, na época da pesquisa. Uma proporção significante de participantes $(16,9 \%)$ pertencia a famílias que ganhavam mais de $R \$ 6.100,00$ por mês ou mais de 12 salários mínimos.

\section{RESULTADOS}

A análise de dados ausentes foi efetuada na amostra original, constatando-se ausência de apenas 0,9\% dos itens de dados. Usando-se o software SPSS $17.0^{\circledR}$, analisou-se o padrão de ausência de dados, constatando-se sua distribuição aleatória completamente ao acaso (teste MCAR de Little: qui-quadrado = 
9076,860; graus de liberdade $=8887$ e $p=0,078$ ), por isso dados ausentes foram substituídos, imputando-se valores médios das respectivas variáveis (HAIR e outros, 2010).

Não se excluíram valores extremos, devido à falta de critérios consistentes para sua identificação, uma vez que nem todas as variáveis tinham distribuição normal e as regras usuais de detecção de outliers uni e multivariados (com base no escore padronizado ou na distância de Mahalanobis) pressupõem normalidade.

Após a análise descritiva dos 697 questionários válidos desta pesquisa, optou-se por dividir aleatoriamente a amostra em duas partes, conforme o processo de validação cruzada, e proceder às análises restantes.

$\mathrm{Na}$ primeira subamostra $(\mathrm{n}=400)$, foram realizadas análises fatoriais exploratórias, com extração de fatores pelo método de componentes principais e rotação varimax. Checaram-se a unidimensionalidade e a confiabilidade das operacionalizações dos construtos do modelo $3 \mathrm{M}$, com a intenção de verificar a necessidade de purificar as escalas usadas, retendose itens não ambíguos e de maior carga fatorial. Foi utilizado o critério de Kaiser (autovalores > 1), para definir a quantidade de fatores extraídos e, para avaliar a qualidade da solução fatorial, variância total extraída de um fator $\geq 60 \%$, exigindo-se soluções fatoriais com teste de esfericidade de Bartlett significativas $(p<0,01)$ e índice KMO (Kaiser-Meyer-Olkin) > o,60 (HAIR e outros, 2010).

Após a exclusão de alguns itens que impediam a unidimensionalidade das escalas e o alcance dos critérios adotados, procedeu-se à análise de confiabilidade dos construtos. Todas as escalas atenderam aos critérios propostos, exceto a escala organização, que teve a variância total extraída <60\% (54\%). Para estimar a consistência interna das medidas, calculou-se o alfa de Cronbach, sendo o valor mínimo aceitável de 0,70, mas podendo ser de apenas de 0,60 , considerando-se a fase exploratória (HAIR e outros, 2010). Após a exclusão de itens, a confiabilidade de todas as escalas foi adequada, exceto a escala organização, que apresentou alfa de Cronbach muito próximo do mínimo aceitável $(0,58)$, sendo os demais alfas superiores a 0,70 (ver Tabela 2).

Após a análise fatorial exploratória, para testar a validade de construto das escalas purificadas, foi realizada uma análise fatorial confirmatória com a segunda subamostra $(n=297)$, utilizando-se o método de estimativa de parâmetros por máxima verossimilhança (KLINE, 2005). A Tabela 1 mostra o resultado dos índices de qualidade do ajuste obtido no modelo de mensuração.

\section{TABELA 1. Índices de qualidade de ajuste do modelo estrutural e de mensuração}

\begin{tabular}{l|c|c|l}
\hline Medidas de ajuste & Modelo de mensuração & Modelo estrutural & Indicação de qualidade do ajuste* \\
\hline Qui-quadrado & 1333,64 & 1533,91 & valor p significante \\
\hline Graus de liberdade (GL) & 794 & 824 & Não se aplica \\
\hline Qui-quadrado/GL & 1,69 & 1,86 & $<2$ (muito bom); entre 2 e 5 (aceitável) \\
\hline valor p & 0,00 & 0,00 & $<0,05$ \\
\hline NFI & 0,81 & 0,78 & Quanto maior, melhor \\
\hline TLI & 0,90 & 0,87 & $>0,90$ \\
\hline CFI & 0,91 & 0,88 & $>0,90$ \\
\hline RMSEA & 0,05 & 0,05 & $<0,07$ se CFI $\geq 0,90$ \\
\hline
\end{tabular}

Fonte: Dados de pesquisa trabalhados no programa AMOS 7.

Nota: $\left(^{*}\right)$ Índices sugeridos por Hair e outros (2010, p. 645, 646 e 686), levando em consideração amostra maior que 250 respondentes e modelo com mais de 30 variáveis observáveis.

No caso de validade convergente, cargas altas em um fator indicam que as cargas convergem para o mesmo fator subjacente. O percentual médio de variância extraída (VE) é um indicador de validade convergente e é calculado como o total de todas as cargas padronizadas dos itens de um construto elevado ao quadrado (correlações múltiplas quadradas) dividido pelo número de itens. Cargas fatoriais significantes, maiores do que o,5, além de $V E \geq 0,5$, sugerem convergência adequada (HAIR e outros, 2010, p. 675). Outro indicador de validade convergente su- gerido pelos autores é a confiabilidade composta (também chama de confiabilidade de construto), que deve ser $\geq 0,70$, ou, pelo menos, entre 0,60 e 0,70 , desde que outros indicadores de validade de construto de um modelo estejam bons. Todos os itens atenderam aos critérios para validade convergente, ou seja, todas as cargas de um item com seu construto foram significativas e > 0,50; todas as escalas apresentaram VE $\geq 0,50$, exceto a escala organização, que apresentou um VE abaixo do recomendado $(0,41)$, e a escala necessidade de recursos corporais, que apre- 
sentou carga muito próxima ao mínimo recomendado $(0,48)$. Todas as escalas apresentaram confiabilidade composta > 0,70, exceto a escala organização, que apresentou um valor também abaixo do recomendado (o,67, porém, como VE também foi baixa, não é possível atribuir validade convergente à escala). Os resultados desses testes encontram-se na Tabela 2.

Validade discriminante é a extensão na qual um construto é diferente de outros. Para ter validade discriminante, um construto deve explicar mais a variância em seus itens de medida do que a variância compartilhada com outros construtos. Ou seja, a variância extraída (VE) de cada construto deve ser maior que a correlação de um item com outros construtos, elevada ao quadrado (HAIR e outros, 2010). Usando tal comparação, todos os construtos apresentaram valores de VE maiores do que os valores da correlação quadrada interconstrutos, indicando que todos os construtos possuem validade discriminante.

Como recomendado por Mowen (2000), todos os oito traços elementares foram incluídos no modelo de pesquisa como variáveis de controle. As relações propostas entre os traços elementares e os traços de outros níveis hierárquicos são baseadas na literatura exposta e em deduções lógicas com base na literatura (ver hipóteses de pesquisa).

\section{TABELA 2. Resultado da análise fatorial exploratória, consistência interna, percentual de variância média extraída e confiabilidade composta, após purificação das escalas}

\begin{tabular}{|c|c|c|c|c|c|c|c|}
\hline Construto & N. itens & КМO & Bartlett & Variância \% & Alfa & VE & Conf. Comp. \\
\hline \multicolumn{8}{|l|}{ Traços elementares } \\
\hline Abertura a experiências & 3 & 0,62 & 0,00 & 69,84 & 0,77 & 0,56 & 0,79 \\
\hline Amabilidade & 3 & 0,65 & 0,00 & 65,38 & 0,73 & 0,52 & 0,76 \\
\hline Necessidade recursos Corporais & 3 & 0,70 & 0,00 & 73,27 & 0,82 & 0,48 & 0,74 \\
\hline Necessidade recursos Materiais & 4 & 0,83 & 0,00 & 76,91 & 0,90 & 0,67 & 0,89 \\
\hline Organização & 4 & 0,60 & 0,00 & 54,26 & 0,58 & 0,41 & 0,67 \\
\hline \multicolumn{8}{|l|}{ Traço composto } \\
\hline Autoestima & 3 & 0,71 & 0,00 & 70,09 & 0,82 & 0,51 & 0,76 \\
\hline \multicolumn{8}{|l|}{ Traços superficiais } \\
\hline Preocupações com a aparência & 4 & 0,72 & 0,00 & 61,05 & 0,78 & 0,50 & 0,80 \\
\hline
\end{tabular}

Notas : 1) Alfa = o valor da estatística alfa de Cronbach; 2) Variância \% = variância total extraída; 3) VE= variância média extraída; 4) Conf. Comp = confiabilidade composta.

Foi construído um modelo estrutural para testar as hipóteses, cujos índices de ajuste do modelo estrutural são mostrados na Tabela 1. No geral, os índices de ajuste do modelo estrutural foram adequados, apesar de CFI $(0,88)$ ter ficado pouco abaixo do limiar recomendado. A Figura 2 mostra o resultado do teste de hipóteses: 
Figura 2. Relações, cargas padronizadas e variância explicada

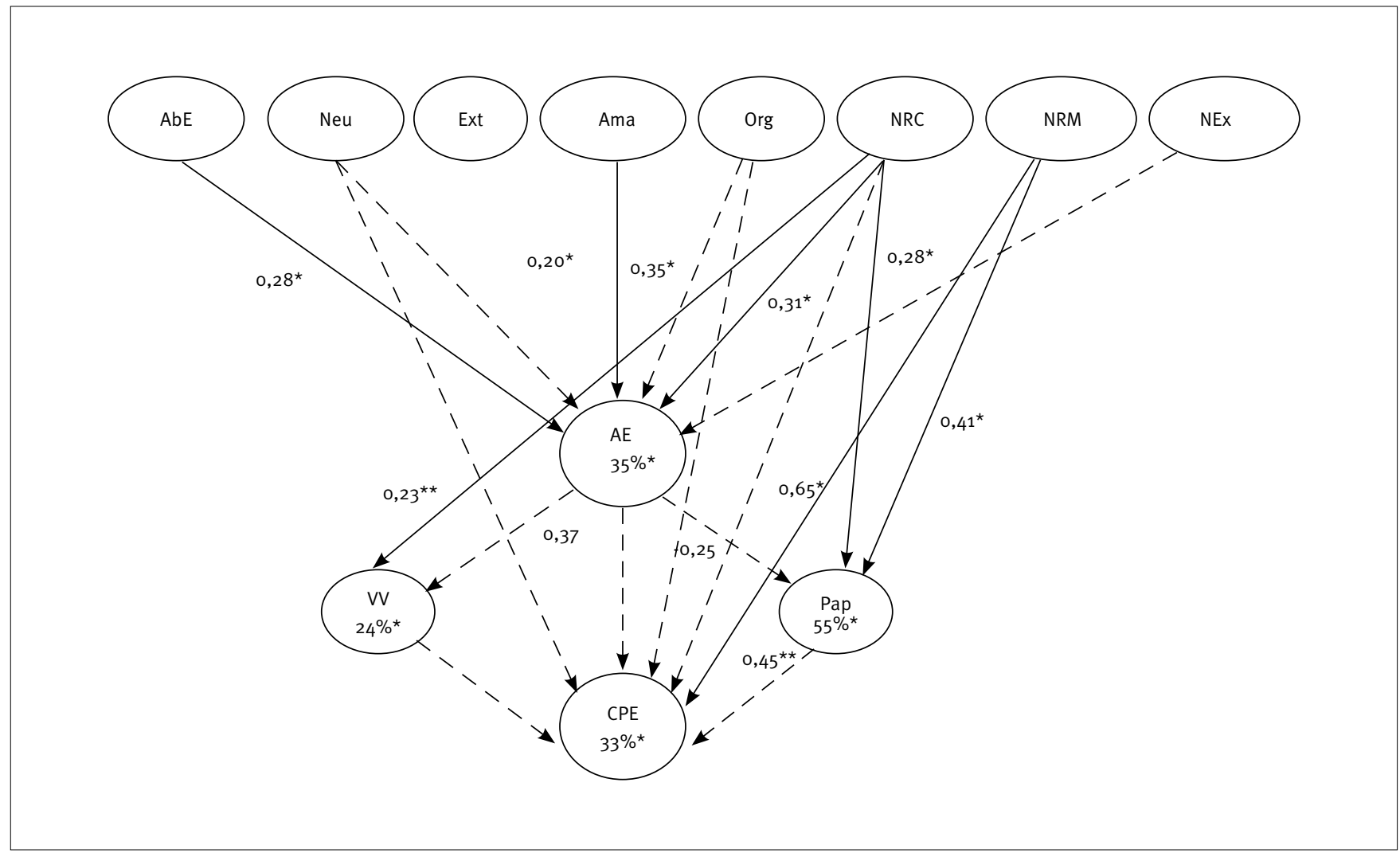

Notas:

1) ${ }^{\star}=$ valor $p<0,01$;

2) ${ }^{\star *}=$ valor $p<0,05$;

3) setas inteiras = hipóteses suportadas;

4) setas tracejadas = hipóteses refutadas;

5) valores em negrito e em percentuais = variância explicada;

6) $\mathrm{AbE}=$ abertura à experiências; Neu = neuroticismo; Ext= extroversão; $\mathrm{Ama}=$ amabilidade; Org = organização; $\mathrm{NRC}=$ necessidade de recursos corporais; $\mathrm{NRM}=$ necessidade de recursos materiais; $\mathrm{NEx}=$ necessidade de excitação; $\mathrm{AE}=$ autoestima; $\mathrm{VV}=$ visão vaidosa;

Pap = preocupação com a aparência e CPE = propensão à cirurgia plástica estética.

0 resultado do teste de hipóteses mostra que os traços elementares correlacionados com autoestima são abertura à experiência, extroversão, amabilidade e necessidade de recursos corporais. Isso significa que quanto mais uma mulher demonstra necessidade de encontrar novas soluções, expressar ideias originais e usar a imaginação na execução de tarefas, é extrovertida, amável e tem necessidade de manter e realçar o corpo, maior será sua autoestima.

Os traços antecedentes de preocupação com a aparência, que é um traço de vaidade, são autoestima, necessidade de recursos materiais e corporais, indicando que mulheres com autoestima baixa, materialistas e com necessidade maior de manter e realçar o corpo são mais preocupadas com a aparência e mais vaidosas que as mulheres que não apresentam tais carac- terísticas. Vale ressaltar que a necessidade de recursos materiais é o traço que mais influencia a preocupação com a aparência.

0 traço visão vaidosa teve como traços antecedentes a autoestima e necessidade de recursos corporais, ou seja, quanto maior a autoestima de uma mulher e quanto mais ela necessita manter e realçar seu corpo, melhor será a avaliação que fará de sua aparência.

Vale ressaltar que, dos dois traços que mensuram vaidade, somente preocupação com aparência parece predizer a propensão à CPE, o que suporta a hipótese 6 e refuta a hipótese 7 . A relação entre autoestima e propensão à CPE também não se confirmou, mostrando que o traço pode afetar indiretamente o desejo de se submeter à uma CPE, por meio da influência na preocupação com a aparência. 


\section{IMPLICAÇÕES TEÓRICAS E GERENCIAIS DOS RESULTADOS}

O principal objetivo do trabalho foi compreender a relação entre autoestima, variáveis de personalidade e motivação na vaidade feminina e como essas variáveis podem influenciar a busca de CPEs. Os resultados mostraram que o traço de vaidade preocupação com aparência influencia a propensão à CPE, um tipo de serviço relacionado à busca pela beleza. Uma contribuição deste estudo refere-se à descoberta de traços que podem predizer níveis de autoestima, vaidade e propensão à CPE em mulheres. Outra contribuição é a validação de escalas para mensurar autoestima, vaidade e propensão à CPE. Análises mostraram que as escalas possuem boa validade e confiabilidade (exceto a escala para medir o traço elementar organização). Embora as conclusões desta pesquisa não possam ser generalizadas para a população brasileira, acredita-se que o estudo tenha contribuído para a compreensão do consumo de CPE, cuja procura teve grande aumento nas últimas décadas.

$\mathrm{Na}$ aplicação do modelo $3 \mathrm{M}$, a propensão feminina de fazer CPE, interpretada como traço superficial, é positivamente correlacionada com a preocupação excessiva com aparência (traço situacional) e necessidade de reunir e possuir bens materiais (traço elementar). Além disso, a preocupação excessiva com aparência associa-se negativamente com autoestima (traço composto) e positivamente com a necessidade de recursos corporais e materiais. A visão vaidosa, ou seja, a preocupação exagerada com a própria aparência, o outro traço utilizado para operacionalizar o construto vaidade, apresentou correlação positiva com autoestima e necessidade de recursos corporais. Por fim, autoestima correlaciona-se positivamente com quatro traços elementares: abertura a experiências, extroversão, amabilidade e necessidade de recursos corporais.

$\mathrm{Na}$ indústria da beleza, esses resultados podem ser aplicados nas decisões estratégicas e na gestão do composto de marketing. Campanhas de comunicação que enfatizem a distância da aparência atual das mulheres em relação aos padrões ideais de beleza feminina podem desafiar sua autoestima, levando-as a aumentar sua preocupação com aparência e, consequentemente, sua disposição de realizar CPEs. Por outro lado, campanhas direcionadas a fortalecer a autoestima das mulheres, desvinculando-a de sua aparência física, podem servir como desestímulo à busca desnecessária por CPEs, bem como incentivar a oferta de outros tipos de serviços destinados à promoção da saúde.

Anúncios e produtos direcionados à necessidade de recursos corporais e materiais provavelmente serão motivadores para mulheres em que esses traços de personalidade são rela- tivamente fortes, no sentido de impulsionar a preocupação excessiva com a própria aparência, o que pode estimular o interesse por submissão à CPE. Por isso, programas que promovam estilos de vida menos materialistas podem levar as mulheres a diminuir seu interesse em se submeterem a CPEs, combatendo o consumismo também nessa área.

Uma das limitações do estudo foi a falta de evidência de validade convergente da escala para medir o traço organização. Para operacionalizar esse construto em pesquisas futuras, sugere-se o uso de outros indicadores. Outra limitação foi a amostra estudada. Estudantes universitários não representam fielmente a população brasileira de consumidores. Foram pesquisados alunos de uma única universidade, sabidamente pertencentes, em sua grande maioria, às classes A e B. Além disso, a amostra foi de conveniência, o que não permite a generalização de seus dados. Apesar de o desenho deste estudo ter sido feito para contemplar apenas mulheres, essa escolha também é uma limitação, pois não é possível detectar diferenças entre gêneros. A idade das respondentes pode, também, ter provocado um viés na interpretação dos dados, pois a grande maioria das respondentes tinha menos de 30 anos. Por fim, o modelo contemplou apenas a propensão à CPE, por ser um procedimento que envolve riscos, dor e alto custo, porém existem vários outros produtos e serviços relacionados à beleza que podem ser investigados, utilizando os traços propostos de personalidade, autoestima e vaidade. Novos estudos devem superar essas limitações e estender resultados a outros segmentos socioeconômicos, para que se possa obter um panorama mais abrangente e confiável sobre os traços que influenciam a autoestima, vaidade e propensão à CPE na população brasileira.

\section{Nota de agradecimento}

Agradecemos ao CNPQ pelo apoio financeiro à pesquisa sobre personalidade e cirurgia plástica estética que deu origem a este artigo. Ao membro do Corpo Editorial Científico responsável pelo processo de avaliação do artigo e aos revisores anônimos da RAE pelas críticas e sugestões.

\section{REFERÊNCIAS}

ABDALA, P. R. Z. Vaidade e consumo: como a vaidade física influencia o comportamento do consumidor. Dissertação de Mestrado. UFRG: Porto Alegre, 2004.

ASKEGAARD, S; GERSTEN, M. C; LANGER, R. The body consumed: reflexivity and cosmetic surgery. Psychology \& Marketing, v. 19, n. 10, p. 793-812, 2002. 
ASSOCIAÇÃO BRASILEIRA DA INDÚSTRIA DE HIGIENE PESSOAL, PERFUMARIA E COSMÉTICOS; AGÊNCIA BRASILEIRA DE DESENVOLVIMENTO INDUSTRIAL, SERVIÇO BRASILEIRO DE APOIO A MICRO E PEQUENAS EMPRESAS. I/ Caderno de Tendência 2010/2011, ano 2, n. 2, p.31, 2010. Disponível em: http://www.abihpec.org.br/conteudo/caderno_tendencias.pdf. Acesso em 04.04.2010.

BASSO, K; ESPARTEL, L. B; SAMPAIO, C. H; PERIN, M. G; ANTONI, V. L. Personalidade e lealdade: proposições de pesquisa com o modelo metateórico de motivação e personalidade. Revista Economia \& Gestão, v. 19, n. 19,p.102-124, 2009.

BAUMAN, Z. Vida para consumo: a transformação das pessoas em mercadoria. Rio de Janeiro: Jorge Zahar, 2008.

BAUMGARTNER, H. J. Toward a personology of the customer. Journal of Consumer Research, v. 29, n. 2, p. 286-292, 2002.

DAVIS, K. A dubious equity: men, women and cosmetic surgery. Body and Society, v. 8, n. 1, p. 49-65, 2002.

EDMONDS, A. No universo da beleza: notas de campo sobre cirurgia plástica no Rio de Janeiro. In: GOLDENBERG, Mirian. Nu \& vestido: dez antropólogos revelam a cultura do corpo carioca. Rio de Janeiro: Record, 2002. p. 189-261.

EDMONDS, A. The poor have the right to be beautiful: cosmetic surgery in neoliberal Brazil. Journal of the Royal Anthropological Institute, v. 13, n. 2, p. 363-381, 2007.

ENGEL, J; BLACKWELL, R; MINIARD, P. Comportamento do consumidor. 8. ed. Rio de Janeiro: LTC, 2000.

ETCOFF, N; ORBACH, S; SCOTT, J; D'AGOSTINO, H. The real truth about beauty: a global report - findings of the global study on women, beauty and well-being. White paper, 2004. Disponivel em: www. therealbeatycampaign.com. Acesso em 12.05.2010.

GIMLIN, D. Cosmetic surgery: beauty as commodity. Qualitative Sociology, v. 23. n. 1, p. 77-98, 2000.

GOLDENBERG, M. Gênero e corpo na cultura brasileira. Psicologia Clínica, v. 17, n. 2, p. $65-80,2005$.

HAIR Jr, J; BLACK, W; BABIN, B; ANDERSON, R. Multivariate data analyis. 7th ed. New York: Pearson Prentice Hall, 2010.

KLINE, R. Principles and practice of structural equation modeling. 2nd ed. NewYork: The Guilford Press, 2005.

MARKEY, C; MARKEY, P. Correlates of young women's interest in obtaining cosmetic surgery. Sex Roles, n. 61, p. 158-166, 2009.

MONTEIRO, P. R. Personalidade e segmentação do mercado de moda: contrastes e perfil de jovens inovadores segundo o gênero. In: ENCONTRO NACIONAL DOS PROGRAMAS DE PÓS-GRADUAÇÃO EM ADMINISTRAÇÃO DA ASSOCIAÇÃO NACIONAL DE PÓS-GRADUAÇÃO E PESQUISA EM ADMINISTRAÇÃO, 32, 2008, Rio de Janeiro. Anais. Rio de Janeiro: ANPAD, 2008. 1 CD.

MONTEIRO, P. R; VEIGA, R. T; GONÇALVES, C. A. Previsão de comportamentos de consumo usando a personalidade. RAE-eletrônica, v. 8, n.
2, Art.13, 2009. Disponível em http://rae.fgv.br/sites/rae.fgv.br/files/ artigos/10.1590_S1676-56482009000200008.pdf. Acesso 12.05.2010.

MOWEN, J. The $3 \mathrm{M}$ model of motivation and personality: theory and empirical applications to consumer behavior. Boston: Kluer Academic Publishers, 2000.

MOWEN, J. Exploring the trait of competitiveness and its consumer behavior consequences. Journal of Consumer Psychology, v. 14, n. 1 e 2, p. 52-63, 2004 .

MOWEN, J; MINOR, M. Comportamento do consumidor. São Paulo: Pearson Prentice Hall, 2003.

MOWEN, J; SUJAN, H. Volunteer behavior: a hierarchical model approach for investigating its trait and functional motive antecedents. Journal of Consumer Psychology, v. 15, n. 2, p. 170-182, 2005.

MOWEN, J; LONGORIA, A; SALLEE, A. Burning and cutting: identifying the traits of individuals with an enduring propensity to tan and to undergo cosmetic surgery. Journal of Consumer Behavior, v. 8, n. 5 , p. 238-251, 2009.

NETEMEYER, R; BURTON, S; LICHTENSTEIN, D. Vanity: traits aspects of vanity. In: BEARDEN, W; NETEMEYER, R. Handbook of marketing scales: multi-item measures for marketing and consumer behavior research. California: Sage, 1995.

RIBEIRO, J. A; VEIGA, R. T. Proposição de uma escala de consumo sustentável. In: ENCONTRO DE MARKETING DA ASSOCIAÇÃO NACIONAL DE PÓS-GRADUAÇÃO E PESQUISA EM ADMINISTRAÇÃO , 4, 2010, Florianópolis. Anais.Florianópolis: EMA, 2010. 1 CD-ROM.

RICCIARDELLI, R; CLOW, K. Men, appearance, and cosmetic surgery: the role of self-esteem and comfort with the body. Canadian Journal of Sociology, v. 34, n. 1, p. 105-134, 2009.

ROMANO, A; NEGREIROS, J; MARTINS, T. Contributos para a validação da escala de autoestima de Rosenberg numa amostra de adolescentes da região interior norte do país. Psicologia, Saúde \& Doenças, v. 8, n. 1, p. 109-116, 2007.

SARWER, D; MAGEE, L; CLARK, V. Physical appearance and cosmetic medical treatments: physiological and socio-cultural influences. Journal of Cosmetic Dermatology, v. 2, n. 1, p. 29-39, 2004.

SHETH, J; MITTAL, B; NEWMAN, B. Comportamento do cliente: indo além comportamento do consumidor. São Paulo: Atlas, 2001.

SOCIEDADE BRASILEIRA DE CIRURGIA PLÁSTICA. Cirurgia plástica no Brasil - 2009. Disponivel em: http://www.cirurgiaplastica.org.br/publico/ index.cfm. Acesso em: 10.02.2010.

STREHLAU, V. I; CLARO, D. P; NETO, S. A. L. Espelho, espelho meu, existe alguém mais vaidosa do que eu? A vaidade feminina e sua influência na autoestima e no consumo de procedimentos estéticos. IN: ENCONTRO DE MARKETING DA ASSOCIAÇÃO NACIONAL DE PÓS-GRADUAÇÃO E PESQUISA EM ADMINISTRAÇÃO, 4, 2010, Florianópolis. Anais. Florianópolis: EMA, 2010. 1 CD-ROM.

SWAMI, V; CHAMORRO-PREMUZIC, T; BRIDGES, S; FURNHAM, A. Acceptance of cosmetic surgery: personality and individual difference predictors. Body Image, n. 6, n. 1, p. 7-13, 2009. 